\title{
Capsule Commentary on Stevens et al., Variation in Inpatient Consultation Among Older Adults in the United States
}

\author{
Karthik Murugiah, MD \\ Center for Outcomes Research and Evaluation, Yale School of Medicine/Yale-New Haven Hospital, New Haven, CT, USA.
}

J Gen Intern Med 31(3):327

DOI: $10.1007 / \mathrm{s} 11606-015-3254-1$

(c) Society of General Internal Medicine 2015

$\mathrm{J}$

ohn Wennberg first reported what he termed "unwarranted geographic variation" in healthcare delivery in the early 1970s. ${ }^{1}$ In that tradition, Stevens et al. report on the variation in inpatient consultation among older adults in the United States. ${ }^{2}$ Consults are common among hospitalized patients and invaluable when needed. However, the decision to obtain a consultation has a strong discretionary component to it. Using Medicare data from 2009-2010, Stevens at al. found that nearly all hospitalizations included at least one consultation, with most admissions averaging nearly three. However, the use of consultation varied markedly, nearly fourfold across quintiles of non-critical-access hospitals. Patient factors, such as acuity, intensive care unit (ICU) admission and in-patient mortality, expectedly predicted consultation use. However, despite accounting for patient characteristics, hospital and regional factors continued to predict consultation use, with more consultations at larger hospitals and those located in the Northeast, and fewer at non-federal public hospitals. The authors conclude that the degree of variation in consultation use observed and the amount of non-patient-driven variation is evidence that this resource is being used inappropriately, with a high likelihood of both overuse and underuse.

These data are convincing evidence of unwarranted variation in consultation use. The authors note that unmeasured variables, such as physician competency, hospital culture, malpractice environment, and financial relationships, may be contributing to this variation. These are all important areas to study, to determine the causes of variation in the use of inpatient consults. In addition, from a quality measurement point of view, further research is needed to study the appropriateness of consultation for specific conditions and the impact of consultation on patient care decisions and outcomes. That variation exists is clear from Steven's data. What is unclear are the consequences of this variation. In the absence of such information, there is little actionable potential for Steven's findings. Given that medical consultation has complex determinants that are difficult to disentangle, there are barriers to using it as a measure of quality. In the end, broader systematic strategies at cost containment such as Medicare's bundled payment initiative may be the means to curb unwarranted variation, including inpatient consultation.

Conflict of Interest: The author has no conflicts of interest with this article.

Corresponding Author: Karthik Murugiah, MD; Center for Outcomes Research and EvaluationYale School of Medicine/Yale-New Haven Hospital, New Haven, CT 06510, USA (e-mail: karthik.murugiah@yale.edu).

\section{REFERENCES}

1. Wennberg J, Gittelsohn A. Small area variations in health care delivery. Science. 1973;182(4117):1102-8.

2. Stevens JP, Nyweide D, Maresh S, Zaslavsky A, Shrank W, Howell MD, Landon BE. Variation in inpatient consultation among older adults in the United States. J Gen Intern Med. (Article SPI \#3216)

This comment refers to the article available at: http://dx.doi.org/10.1007/ s11606-015-3216-7.

Published online March 6, 2015 